\title{
Exposure to Hypobaric Hypoxia and Reoxygenation Induces Transient Anxiety-Like Behavior in Rat
}

\author{
Iswar Baitharu ${ }^{1,2}$, Vishal Jain ${ }^{2}$, Satya Narayan Deep ${ }^{2}$, Gaurav Kumar ${ }^{2}$, Govindasamy Ilavazhagan ${ }^{3 *}$ \\ ${ }^{1}$ Guru Ghasidas Vishwavidyalaya, Bilaspur, India \\ ${ }^{2}$ Defence Institute of Physiology and Allied Sciences, Defence Research Development Organisation, New Delhi, India \\ ${ }^{3}$ Hindustan University, Chennai, India \\ Email: "govindasamyilavazhagan@gmail.com
}

Received October 30, 2013; revised November 29, 2013; accepted December 8, 2013

Copyright (C) 2013 Iswar Baitharu et al. This is an open access article distributed under the Creative Commons Attribution License, which permits unrestricted use, distribution, and reproduction in any medium, provided the original work is properly cited.

\begin{abstract}
Chronic exposure to hypobaric hypoxia $(\mathrm{HH})$ causes memory impairment and prolonged state of mental confusion. However, effect of high altitude exposure on mood state and its underlying mechanisms have been poorly studied. Present study was undertaken to investigate the mood state alteration following chronic exposure to $\mathrm{HH}$. Male Sprague Dawley rats were divided into five groups and exposed to hypoxia for 3,7,14 and 21 days in an animal decompression chamber at an altitude of 25,000ft. Anxiety- and depression-like behaviors were assessed by using various mazes along with changes in serotonin and glutamate level. Our study revealed a decrease in exploratory, grooming and rearing behavior in open field test following initial exposure to HH for 7 days without affecting the locomotory behavior. Initial exposure to HH-decreased time spent in open arm of elevated plus maze indicating induction of anxiety-like behavior which normalized on prolonged hypoxic exposure for 21 days. Hypoxic exposure for 7 days induced anhedonia and increased despair behavior in rat while there was steady improvement in these behaviors when exposed for 21 days. Decrease in serotonin level was noted in hippocampus along with elevated corticosterone and glutamate level which gradually decreased on prolonged exposure to $\mathrm{HH}$. These findings suggest that initial exposure to $\mathrm{HH}$ increases transient anxiety-like behavior in rats followed by gradual improvement in mood state on prolonged exposure. Further, the study also indicates the involvement of serotonergic system in mood state alteration at high altitude following chronic exposure and reoxygenation.
\end{abstract}

Keywords: Mood State; Anhedonia; Anxiety-Like Behaviors; Hypobaric Hypoxia; Depression; Serotonin

\section{Introduction}

A significant portion of the world population resides at high altitude regions. Apart from natives, natural beauty of the snow-clad peaks attracts a huge population of tourists every year to explore the high altitude. High altitude regions are also important for its strategic locations, for sojourners, mountaineers and defense personnel. All of them face extreme climatic conditions like severe cold, high wind velocity, UV irradiation and most importantly, hypobaric hypoxia due to reduced partial pressure of oxygen. Exposure to $\mathrm{HH}$ is known to cause adaptive physiological changes that enable the organisms to cope up with severity of this environmental stress. However, mal-acclimatisation to extreme altitudes results in many pathological manifestations like High Altitude Pulmonary

"Corresponding author.
Edema (HAPE), High Altitude Cerebral Edema (HACE) and Acute Mountain Sickness (AMS) [1,2]. The stress has also reportedly been associated with sensory motor dysfunction [3] and loss of memory function on prolonged exposure [4,5]. Nausea, vomiting, hypophagia [6], dizziness and insomnia are the other problems encountered at high altitude. However, mental dysfunctions like the induction of prolonged state of confusion [7] and cognitive deficit on exposure to extreme altitude compel human to compromise with their performance that demands higher order mental functioning.

The mechanisms underlying cognitive dysfunctions at high altitude have been attributed to alteration in neurotransmitters synthesis, secretion and metabolism, induction of glutamate excitotoxicity and/or calcium overloading leading to neuronal death $[8,9]$. Recent works in our laboratory show alterations of monoaminergic sys- 
tem in brain regions like striatum, pre frontal cortex and hippocampus following chronic exposure to HH (Unpublished data). While depletion in the level of serotonin, norepinephrine and/or dopamine in hippocampus have been known to be associated with induction of depressive symptoms [10,11], antidepressant elevates these neurotransmitters in the brain alleviate mood states $[10,12]$.

In addition to dopaminergic alteration, HPA axis dysregulation plays a major role in the pathophysiology of anxiety and depression. Hypothalamic corticosteroid releasing factor overexpression contributes to HPA axis hyperactivity in psychiatric patients [13]. Persistent elevation in corticosterone level following prolonged exposure to $\mathrm{HH}$ leads to augmented neurodegeneration and memory impairment $[14,15]$. Apart from HPA axis hyperactivity, altered glutamatergic neurotransmission has been a major pathological factor in depression and glutamatergic agents have demonstrated rapid and robust antidepressant activity in humans. Because of intrinsic connection of glutamate receptors to neuronal efficiency and inefficiency cascades, glutamatergic dysfunctions lead to alterations of multiple signal transduction pathways crucial for neuronal survivability [16]. Chronic exposure to $\mathrm{HH}$ has been reported to cause neurodegeneration in hippocampal region through elevated glutamate excitotoxicity [17].

Since chronic exposure to $\mathrm{HH}$ is known to cause neuronal death in a duration dependent manner and consequently leads to impairment in the consolidation as well as retrieval of memory, it may also influence the mood state as evident from the increased occurrence of mental confusion and headache. HH-induced loss of memory could further aggravate the behavioral alteration and precipitate occurrence of anxiety-like behavior. In the present study, we evaluated the behavioral alteration in rats following chronic exposure to HH. Temporal alteration in serotonin and glutamate level following exposure to $\mathrm{HH}$ are also evaluated to find out the correlation between modulated neurotransmitters level in central nervous system and ensuing behavioral alteration following hypoxic exposure.

\section{Materials and Methods}

\subsection{Chemicals and Reagents}

All the chemicals and reagents were procured from Sigma-Aldrich (USA) if otherwise mentioned. HPLC standards for glutamate and serotonin were purchased from Sigma-Aldrich chemicals (Sigma-Aldrich chemicals, USA). Kits for estimation of serotonin was procured from GenWay Biotech Inc. (USA).

\subsection{Animals}

All the experimental protocols followed were approved by the ethical committee of the institute following the guidelines of "Committee for the Purpose of Control and Supervision of Experiments on Animals," Govt. of India. Adult male Sprague Dawley rats weighing 220 - 230 g (3 month old) were taken and maintained at 12 hour light and dark cycle in animal house of the institute. Food pellets (Lipton Pvt Ltd, India) and water was given ad libitum. The temperature and humidity of the animal house was maintained at $28^{\circ} \mathrm{C} \pm 2^{\circ} \mathrm{C}$ and $55 \%-60 \%$ respectively. All animal handling was performed between a time windows of $10.00 \mathrm{~h}$ to $12.30 \mathrm{~h}$ to avoid experimental discrepancy arising due to diurnal variations in corticosterone concentration.

\subsection{Experimental Design}

Rats $(n=60)$ were handled everyday by the same person for $5 \mathrm{~min}$ each to habituate properly for 15 days prior to behavioral tests. Rats $(n=12$ /group) were randomly divided into five groups and exposed to simulated hypobaric hypoxia in a decompression chamber for stipulated period of time. While group I served as normoxic group, rest four groups of rats were designated as 3 days hypoxic, 7 days hypoxic, 14 days hypoxic and 21 days hypoxic group. Behavioral tests were performed immediately after the hypoxic exposure.

\subsection{Exposure to Simulated HH}

Animals to be exposed to $\mathrm{HH}$ were inducted to a simulated altitude of $7600 \mathrm{~m}(25,000 \mathrm{ft}, 282 \mathrm{~mm} \mathrm{Hg})$ in a specially designed animal decompression chamber where altitude could be maintained by reducing the ambient barometric pressure. Fresh air was continuously flushed at a rate of $8 \mathrm{~L} / \mathrm{min}$ to prevent accumulation of carbon dioxide within the chamber. The temperature and humidity in the chamber were maintained precisely at $27^{\circ} \mathrm{C} \pm$ $2{ }^{\circ} \mathrm{C}$ and $63 \% \pm 2 \%$ respectively. The rate of ascent and descent to hypobaric conditions was maintained at 300 $\mathrm{m} / \mathrm{min}$. The hypobaric hypoxic exposure was continuous for the stipulated period excepting for a $15-20 \mathrm{~min}$ interval each day for replenishment of food and water and changing the cages housing the animals.

\subsection{Sample Preparation and Analysis}

After scheduled period of exposure to $\mathrm{HH}$ in a simulated decompression chamber, behavioural assessment was performed. The sampling of the plasma and hippocampus was done between 9:30 AM - 10:30 AM for normoxic and all other hypoxia exposed group immediately after completion of behavioral test to avoid the circadian rhythm mediated variation in corticosterone level .The rats were then sacrificed by cervical dislocation and brain was isolated at $4^{\circ} \mathrm{C}$ immediately. The hippocampi were isolated and snap freezed to prevent degradation of neu- 
rotransmitters and other unstable compounds. The samples were then stored at $-80^{\circ} \mathrm{C}$ till the time of analysis. The samples were homogenised with the help of polytron homogeniser in respective buffer or solution as per manufacturer protocol provided in the kits.

\subsection{Behavioral Assessment}

\subsubsection{Elevated Plus Maze}

The test setting consisted of a plus-shaped apparatus with two open and two enclosed arms, each with an open roof, elevated $40-70 \mathrm{~cm}$ from the floor. Rats were placed at the junction of the open and closed arms, facing the open arm opposite to the experimenter. The video-tracking system automatically recorded the number of entries and time spent by the rat in the open and closed arms using the ANY maze software (Stoelting Co). At the end of 5-min test, rats were removed from the plus maze and placed in a transport cage. Elevated plus maze was cleaned with alcohol and dried with paper towels before testing with another rat. Reduction in anxiety level was indicated by an increase in the proportion of time spent in the open arms (time in open arms/total time in open or closed arms), and an increase in the proportion of entries into the open arms (entries into open arms/total entries into open or closed arms). Total number of arm entries and number of closed-arm entries are usually employed as measures of general activity.

\subsubsection{Open Field Test}

Open field test was used to assess the locomotory as well as the exploratory behavior of rats. The open field maze was divided into two zones, central and peripheral zone, using the square drawn on the maze. The apparatus consisted of a rectangular area of $81 \times 81 \mathrm{~cm}$ surrounded by a $28 \mathrm{~cm}$ high wall. The area was divided into 16 squares of $20 \times 20 \mathrm{~cm}$ by painted white lines. The field was lighted with a $40 \mathrm{~W}$ bulb fixed $50 \mathrm{~cm}$ above the field. Light was focused on the center of the field with the periphery remaining dark. The rat was placed in one corner of the open field and its activity during the subsequent 5 min was assessed. Horizontal locomotion (number of times crossings of the white lines), frequency of rearing or leaning (sometimes termed vertical activity), frequency of grooming (protracted washing of the coat), defecation (number of fecal pellets) and time spent in peripheral and central zone were observed and recorded using ANY Maze software (Stoelting Co).

\subsubsection{Rota Rod Test}

The rotating rod apparatus was used to assess motor performance and measure the ability of rat to improve motor skill performance with training. Rats were placed on the elevated accelerating rod (rota rod, $3 \mathrm{~cm}$ in diameter) beginning at $5 \mathrm{rpm} / \mathrm{min}$ for four trials per day for three consecutive days. Each trial lasted a maximum of $10 \mathrm{~min}$, during which time the rotating rod underwent a linear acceleration from 4 to $40 \mathrm{rpm}$ over the first $5 \mathrm{~min}$ of the trial and then remained at maximum speed for the remaining $5 \mathrm{~min}$. Animals were scored for their latency (in seconds) to fall (height $20 \mathrm{~cm}$ ) for each trial. Animals rested a minimum of $10 \mathrm{~min}$ between trials to avoid fatigue.

\subsubsection{Sucrose Preference Test}

Rats were trained to take water/water containing sucrose from two bottles [18]. The consumption of water was assessed for 2 days before the test. After a $12 \mathrm{~h}$ period (between 10 and $12 \mathrm{~h}$ of the light phase) of water deprivation, the sucrose preference test was performed following exposure to different duration of $\mathrm{HH}$ till 21 days, with a free choice between two droppers of $15 \mathrm{ml}$ placed in each cage, one containing sucrose ( $2 \%$ in water) and the other containing water. To eliminate potential side preferences, the position of the droppers was switched after 2 days. The consumption of water and sucrose solution was assessed daily. The preference for sucrose was calculated as the volume of sucrose solution consumed relative to the total volume of liquid consumed and expressed in percentage change taking control value as $100 \%$.

\subsubsection{Forced Swim Test}

The forced swim test (FST), a standardized test of depression-like behavior in which depression is inferred from increased duration of immobility, was conducted as described previously [19]. The duration of immobility was determined during the test using the mobility function of ANY maze (Stoelting Co). Briefly, rats were plunged individually into a vertical Plexiglas cylinder (25 $\mathrm{cm}$ high; $10 \mathrm{~cm}$ in diameter) filled with 9-cm-deep water $\left(21^{\circ} \mathrm{C}-23^{\circ} \mathrm{C}\right)$. After the 6 -min period of the test, they were removed and allowed to dry. Immobility (i.e., making only minimal movements to keep the head above water or floating) was measured for two 3-min periods by an observer blind to the condition of the rats. Swimming time and struggling was also scored to know the active coping style in rats.

\subsection{Assessment of Stress Marker and Neurotransmitters}

\subsubsection{Estimation of Corticosterone by HPLC}

Levels of corticosterone was estimated both in plasma and hippocampal tissue using high performance liquid chromatography (Waters, Milford, MS, USA). The extraction of corticosterone from plasma and hippocampal tissue was done with methylene chloride and diethyl ether respectively [20,21]. The ether and methylene chloride evaporated tissue and plasma samples were recon- 
stituted with $250 \mu 1$ of methanol. $10 \mu 1$ of the reconstituted sample was injected with the help of an auto sampler (Waters) to the HPLC system and resolved using $\mathrm{C}_{18}$ RP column with acetonitrile:Water:Glacial acetic acid $(35: 65: 05 \mathrm{v} / \mathrm{v})$ as solvent phase in isocratic condition. The flow rate of the mobile phase was maintained at 1 $\mathrm{ml} / \mathrm{min}$ and detection of corticosterone fraction was done at $254 \mathrm{~nm}$ with a UV detector. The pressure in the column was maintained at $1800 \mathrm{psi}$ and the samples were run for 30 minutes. A standard plot was prepared using the endogenous steroid free plasma and methanol for plasma and hippocampal tissue sample respectively in the range of $10-1000 \mathrm{ng} / \mathrm{ml}$ by serial dilution. The standards were tested individually at different concentrations to record detection limit, retention time and peak area. Concentration of corticosterone was calculated from a standard plot of peak area of corticosterone versus concentration of corticosterone.

\subsubsection{Serotonin Level in Hippocampus}

The level of serotonin was estimated using Serotonin Elisa kit from tissue homogenate immediately after isolation of the hippocampal region as per manufacturer protocol. Briefly, standard, acylated control and acylated sample was pipetted into the respective wells of the microtiter plate. Serotonin biotin was added to each well followed by addition of serotonin antiserum. After 90 min of incubation at room temperature on an orbital shaker, the plate was washed thrice with diluted wash buffer followed by addition of freshly prepared enzyme conjugate. The plate was then covered and incubated for $60 \mathrm{~min}$ at room temperature followed by washing thrice with diluted wash buffer. Freshly prepared PNPP substrate solution was then added into each well and incubated $60 \mathrm{~min}$ at room temperature. The reaction was then stopped by adding PNPP stop solution into each well. Optical density was measured with Elisa plate reader at $405 \mathrm{~nm}$ within $60 \mathrm{~min}$. The concentration of serotonin was estimated using standard plot. Final concentration of serotonin in the tissue sample was calculated by multiplying with dilution factor and expressed in $\mathrm{ng} / \mathrm{mg}$ tissue.

\subsubsection{Estimation of Glutamate by HPLC}

Reverse phase HPLC was performed taking L-glutamate as standard using $\mathrm{C}_{18} \mathrm{RP}$ column and fluorescent detector. Column was equilibrated with mobile phase $(0.05 \mathrm{M}$ sodium acetate buffer, $\mathrm{pH} 7.0$, with $26 \%$ methanol) at a flow rate of $0.5 \mathrm{ml} / \mathrm{min}$ and pressure of $10 \mathrm{~kg} / \mathrm{cm}^{2}$ for 1 $\mathrm{hr}$. Derivatisation stock solution was prepared by adding $2 \mathrm{mg}$ o-phthaldehyde (OPA), $1 \mathrm{ml}$ methanol and $0.07 \mathrm{ml}$ $\beta$-mercaptoethanol which was then diluted ten times to be used as working solution. $50 \mu 1$ hippocampal sample was derivatised in derivatisation solution $(0.05 \mathrm{M}$ sodium acetate, $\mathrm{pH}$ 9.0, OPA) for $3 \mathrm{~min}$, filtered with $0.2 \mu \mathrm{m}$ cellulose acetate filters and injected to the column along with standard. The flow rate of mobile phase was maintained at $1 \mathrm{ml} / \mathrm{min}$ and excitation and emission wavelength was set at $330 \mathrm{~nm}$ and $450 \mathrm{~nm}$ respectively. Analysis and quantification was done using the standard plot chromatogram peak area.

\section{Statistical Analysis}

The means and standard deviations of each set of rats i.e. normoxia, 3 days, 7 days, 14 days and 21 days HH were calculated. Statistical comparison was done between control and hypoxic groups. The post hoc analysis was done by Newman-Keul's test in all experimental groups wherever appropriate by using one way analysis of variance (ANOVA). Difference below or equal to the probability level 0.05 was considered statistically significant.

\section{Results}

\subsection{Elevated Plus Maze}

Exposure to $\mathrm{HH}$ for 3 and 7 days significantly decreased the time spent in open arm with corresponding increase in time spent in closed arm compared to normoxic group. Prolonged exposure to $\mathrm{HH}$ for 21 days, however, significantly increased the time spent in open arm and was comparable to normoxic group as shown in Figures 1(a) and (b). Number of entries to the open arm decreased significantly on 3 days and 7 days of hypoxic exposure followed by a gradual increase with increase in duration of exposure to $\mathrm{HH}$. However, there was no significant change in the closed arm entries following exposure to $\mathrm{HH}$. Prolonged exposure to $\mathrm{HH}$ significantly increased the number entries to open arm and was comparable to normoxic group as shown in Figures 1(a) and (b).

\subsection{Open Field Test}

There was significant decrease in the grooming, rearing and locomotory behavior following exposure to $\mathrm{HH}$ for 3 and 7 days compared to normoxic group. However, following prolonged exposure to $\mathrm{HH}$ for 21 days, grooming, rearing and locomotory behavior of rats significantly increased compared to 3 days and 7 days hypoxic group and were comparable to normoxic group as shown in Figures 2(b) and (c). Defecation was significantly increased when exposed to hypoxia for 7 days compared to normoxic group and gradually decreases on prolonged exposure to HH for 21 days (Figure 2(b)). Exposure to $\mathrm{HH}$ for 3 and 7 days significantly decreased the time spent in central zone with with corresponding increase in the time spent in the peripheral zone compared to the normoxic group. Prolonged exposure to $\mathrm{HH}$, in turn, significantly increased the time spent in central zone and decreased the time in peripheral zone compared to 7 days 

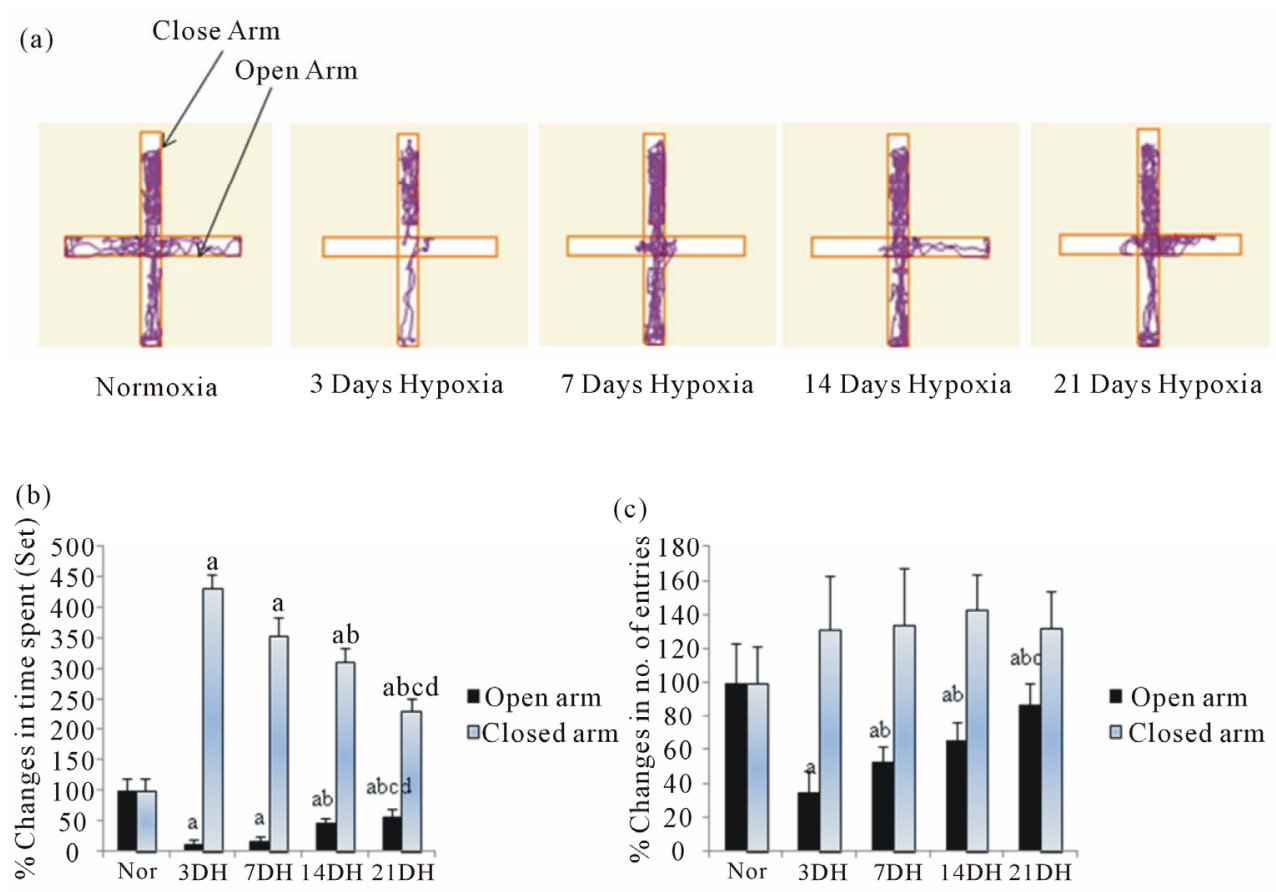

Figure 1. (a). Representative track sheets showing duration dependent changes in the time spent and number of entries to the open arm and closed arm in Elevated Plus Maze. (b) Changes in time spent in open arm and closed arm in Elevated Plus Maze following exposure to HH. (c). Changes in number of entries to open and closed arm following exposure to HH. "a" denotes $p<0.05$ vs when compared with normoxia, "b" denotes $p<0.05$ vs when compared with 3 days hypoxia, "c" denotes $p<0.05$ Vs when compared to 7 days hypoxia and " $d$ " denotes $p<0.05$ Vs when compared with 14 days hypoxia.

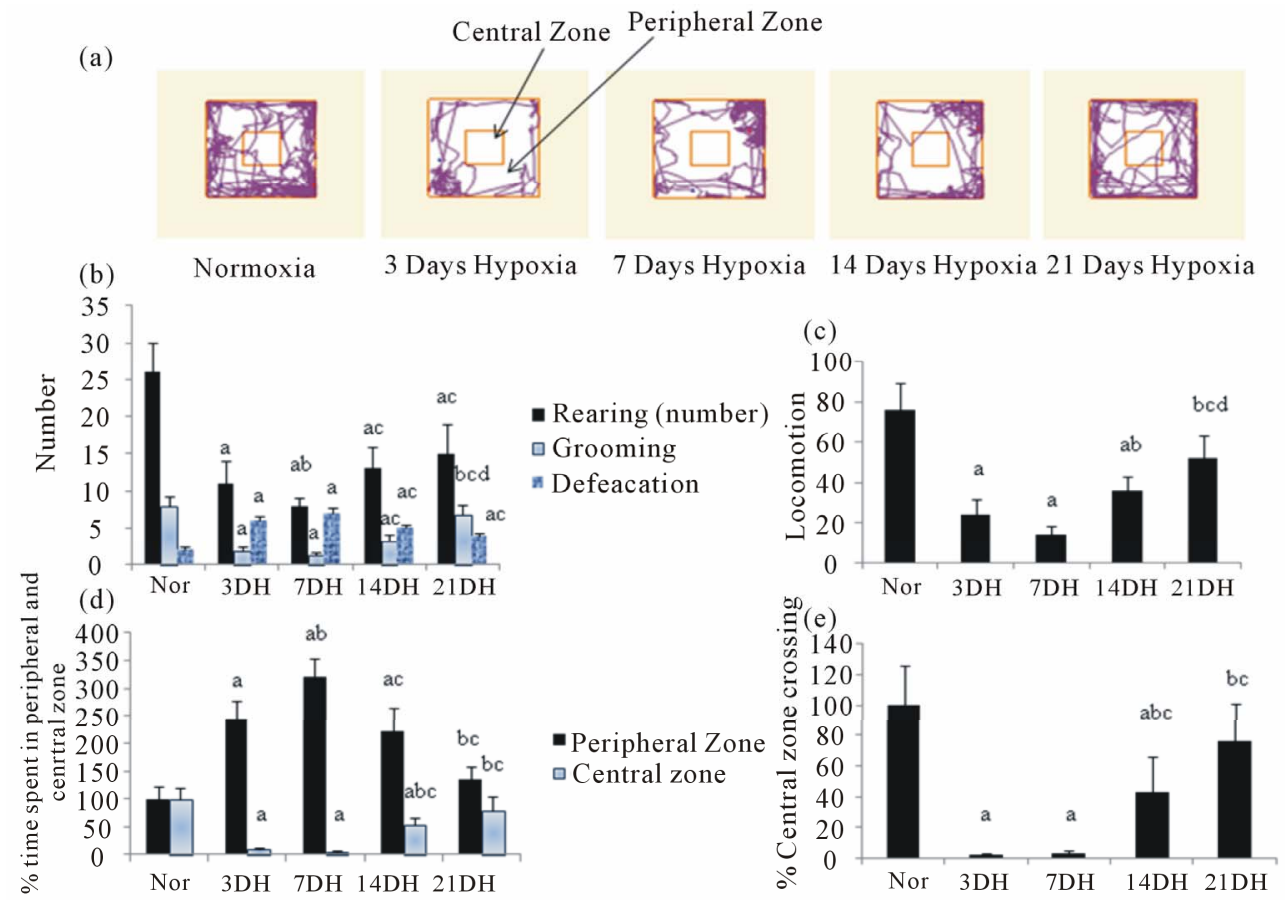

Figure 2. (a). Representative track sheets showing alteration in locomotion and exploratory behavior in open field test (distance travelled in central zone vs peripheral zone). (b). Changes in grooming, rearing and defecation behavior of rats following exposure to HH. (c). Changes in the locomotory behavior following exposure to HH. (d). Changes in the time spent in peripheral and central zone of open field and (e). Changes in the central zone crossing following exposure to HH. "a" denotes $p<0.05$ vs when compared with normoxia, "b" denotes $p<0.05$ vs when compared with 3 days hypoxia, "c" denotes $p<0.05$ vs when compared to 7 days hypoxia and "d" denotes $p<0.05$ vs when compared with 14 days hypoxia. 
hypoxic group as shown in Figure 2(d). There was significant decrease in central zone crossing following exposure to $\mathrm{HH}$ for 3 and 7 days compared to normoxic group which gradually increased significantly on prolonged hypoxic exposure (Figure 2(e)).

\subsection{Sucrose Preference Test}

Sucrose preference (Volume of sucrose solution consumed/Total liquid consumed (water + sucrose solution) was significantly decreased when exposed to $\mathrm{HH}$ for 3 and 7 days compared to normoxic group. Though there was a gradual increase in sucrose intake on prolonged exposure for 21 days, it was significantly low compared to normoxic group as shown in Figure 3(a).

\subsection{Rota Rod Test}

No significant change in the latency of fall was noted in any group exposed to $\mathrm{HH}$ for different durations compared to normoxic group (Figure 3(b)).

\subsection{Forced Swim Test}

There was significant increase in the immobility time in 3 and 7 days of exposure to $\mathrm{HH}$ compared to normoxic group. Prolonged exposure for 21 days, however, decreased the immobility time significantly compared to 3 and 7 days hypoxic group and was comparable to normoxic group though the value still remain higher then normoxic value as shown in Figure 3(c). On the other hand, swimming time significantly decreased with corresponding increase in struggling time following exposure to $\mathrm{HH}$ for 7 days compared to normoxic group followed by gradual increase in swimming time and decrease in struggling time on prolonged hypoxic exposure for 21 days as shown in Figure 3(c).

\subsection{Alteration in Neurotransmitters and Stress Marker}

\subsubsection{Serotonin Level}

Serotonin level in hippocampus decreased significantly on exposure to $\mathrm{HH}$ for 3 and 7 days compared to normoxic group. However, on prolonged exposure for 14 and 21 days, there was no significant difference in serotonin level compared to normoxic group as shown in $\mathbf{F i -}$ gure 3(d).

\subsubsection{Changes in the Level of Corticosterone}

The corticosterone level in hippocampus as estimated by the HPLC showed significant increase on initial days of exposure to $\mathrm{HH}$ for a period of 3 and 7 day compared to normoxic group. Corticosterone level, however, decreases marginally on 14 days of exposure to $\mathrm{HH}$ though the values were still higher than the normoxic group as shown in Figures 4(a) and (b). There was a corresponding progressive increase in the plasma corticosterone level following 3 and 7 days of exposure to $\mathrm{HH}$ when compared to the normoxic group. Chronic exposure for 14 days, on the other hand, decreased the plasma corticosterone level compared to the 7 days hypoxic group. Plasma corticosterone levels in the 14 days exposure group were significantly above the normoxic and 3 days hypoxic group. Exposure to $\mathrm{HH}$ for 21 days decreased the plasma corticosterone level compared to 3, 7 and 14 days hypoxic group and was comparable to normoxic levels as shown in Figure 4(c).

\subsubsection{Alteration of Glutamate Level}

The glutamate level in hippocampus showed significant increase on initial days of exposure to $\mathrm{HH}$ for a period of 3 and 7 days compared to normoxic group. Glutamate level, however, decreases on 14 days of exposure to $\mathrm{HH}$ though the values were still higher than the normoxic group as shown in Figures 5(a) and (b).

\section{Discussion}

Stress is one of the most important etiological factors in psychiatric disorders, especially in anxiety and depression. Depressive disorders are marked by chronic depressed mood, inability to experience pleasure, withdrawal of interest, feelings of worthlessness and suicidal tendencies $[22,23]$. Recent studies shows that stress induced disturbance in cognitive processes, especially impairment of learning and memory functions accelerate onset and development of depressive mood state [24,25]. However, the underlying mechanisms are not clearly understood. Present study reports that chronic exposure to $\mathrm{HH}$ induces transient anxiety-like behavior in rats. The study further indicates the involvement of serotonergic neurons in hypoxia induced anxiety-like behavior.

Behavioral assessment of rats following exposure to $\mathrm{HH}$ using elevated plus maze, in the present study, showed a gradual decrease in time spent as well as distance covered in open arm indicating induction of anxiety-like behavior in rats. There was no change in the number of closed arm entries following exposure to $\mathrm{HH}$ in elevated plus maze test. However, the reduction in number of entries to open arm and distance covered in both open and closed arm could be due to immobility of rats caused by impaired locomotory activities. Further, we performed open field test as well as rota rod test to find out if there was any locomotory impairment on hypoxic exposure. Open field test showed increased activity in the peripheral region whereas the time and distance travelled in the central region was significantly decreased in hypoxia exposed group compared to normoxic group [26]. The open field test through light on the fact that the chronic exposure to $\mathrm{HH}$ doesnot affect the locomotory 

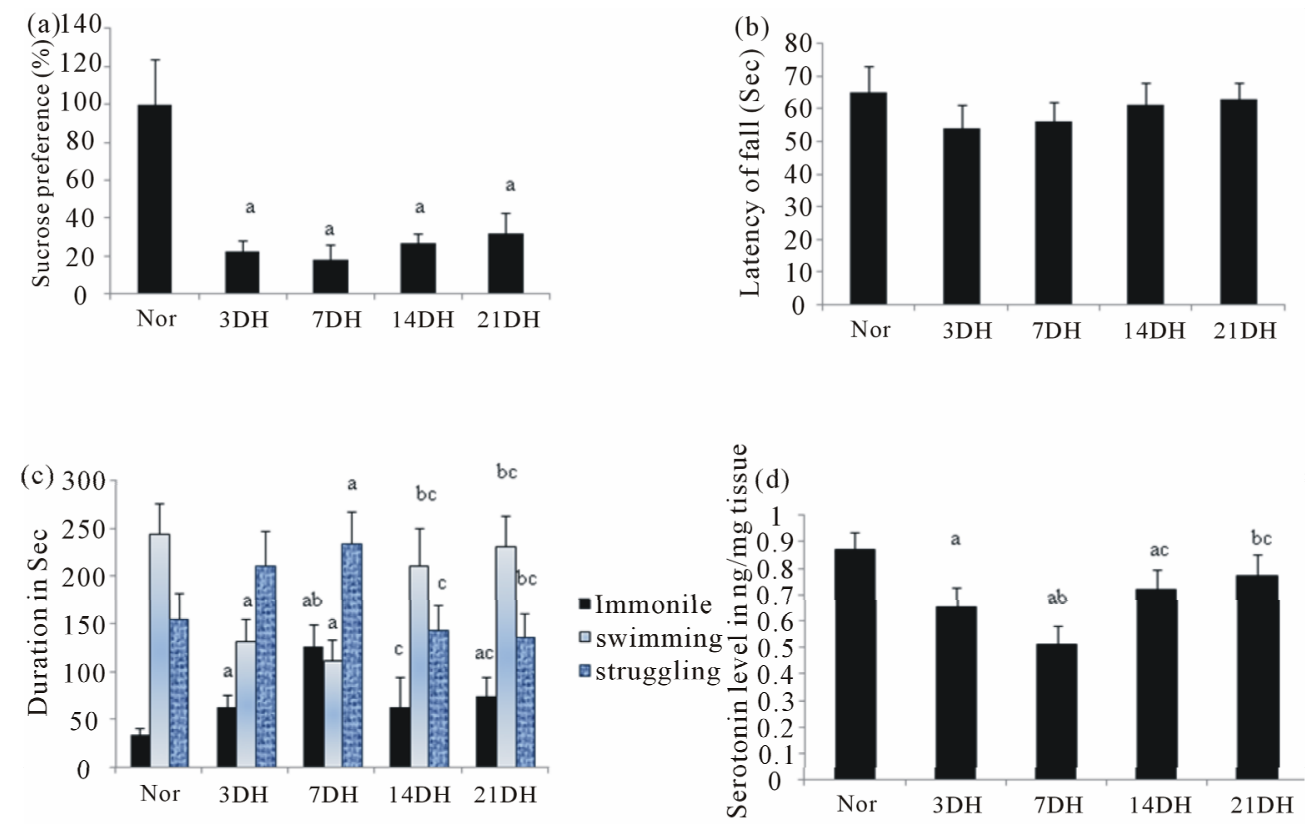

Figure 3. (a). Sucrose intake decreases drastically on initial exposure to $\mathrm{HH}$ followed by a progressive increase on prolonged exposure for 21 days. (b). No significant changes in latency of fall in rota rod test on exposure to HH for different duration. (c). Changes in the immobility, swimming and struggling in forced swim test following exposure to $\mathrm{HH}$. (d). Changes in the level of serotonin in hippocampal tissue following exposure to different duration of $\mathrm{HH}$. "a" denotes $p<0.05$ vs when compared with normoxia, "b" denotes $p<0.05$ vs when compared with 3 days hypoxia, "c" denotes $p<0.05$ Vs when compared to 7 days hypoxia and "d" denotes $p<0.05$ Vs when compared with 14 days hypoxia. "a" denotes $p<0.05$ vs when compared with normoxia, "b" denotes $p<0.05$ vs when compared with 3 days hypoxia, "c" denotes $p<0.05$ Vs when compared to 7 days hypoxia and "d" denotes $p<0.05$ Vs when compared with 14 days hypoxia.
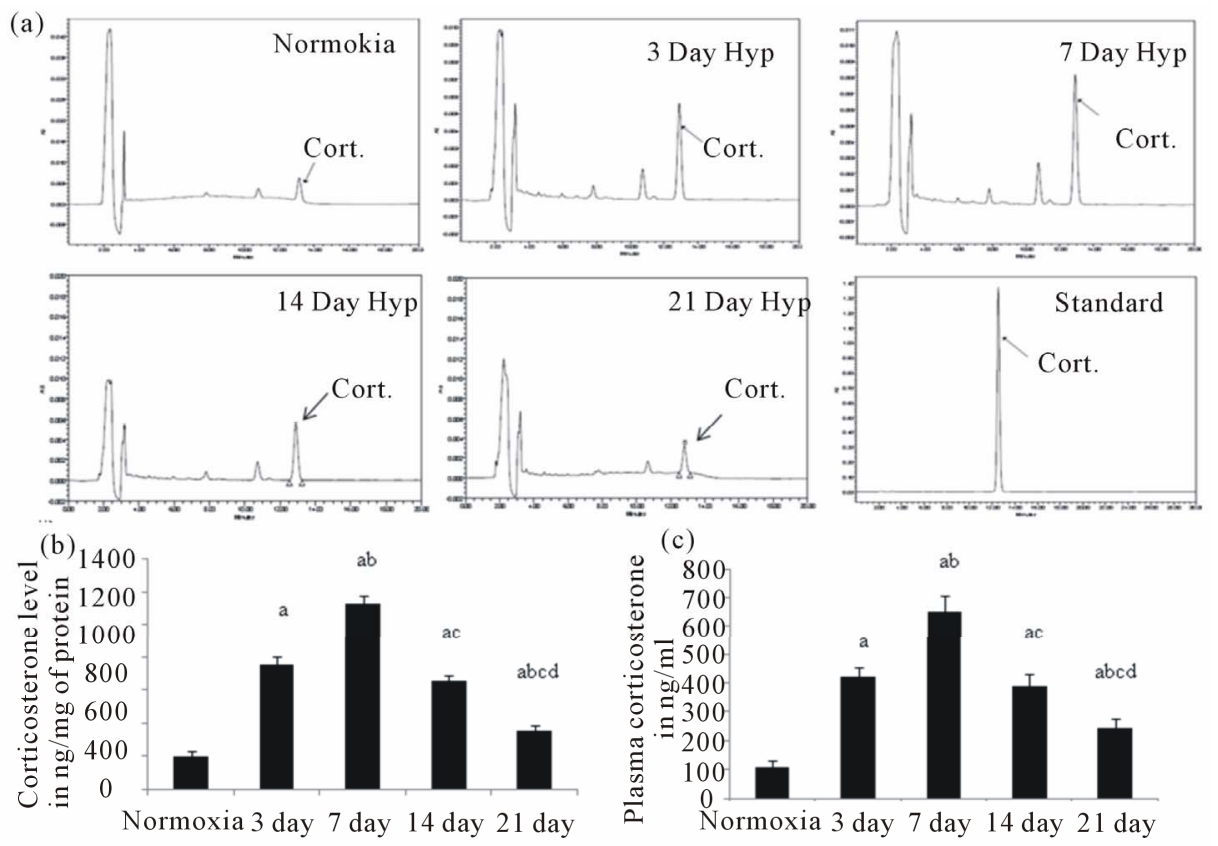

Figure 4. Representative HPLC Chromatograms showing peak for corticosterone. (a) The peak for corticosterone of standard, normoxic group, 3 day hypoxic group, 7 days hypoxic group, 14 days hypoxic group and 21 days hypoxic group. (b) Changes in the level of corticosterone in hippocampus following exposure to $\mathrm{HH}$. (c) Changes in the level of plasma corticosterone following exposure to HH. "a" denotes $p<0.05$ vs when compared with normoxia, "b" denotes $p<0.05$ vs when compared with 3 days hypoxia, "c" denotes $p<0.05$ Vs when compared to 7 days hypoxia and "d" denotes $p<0.05$ Vs when compared with 14 days hypoxia. 

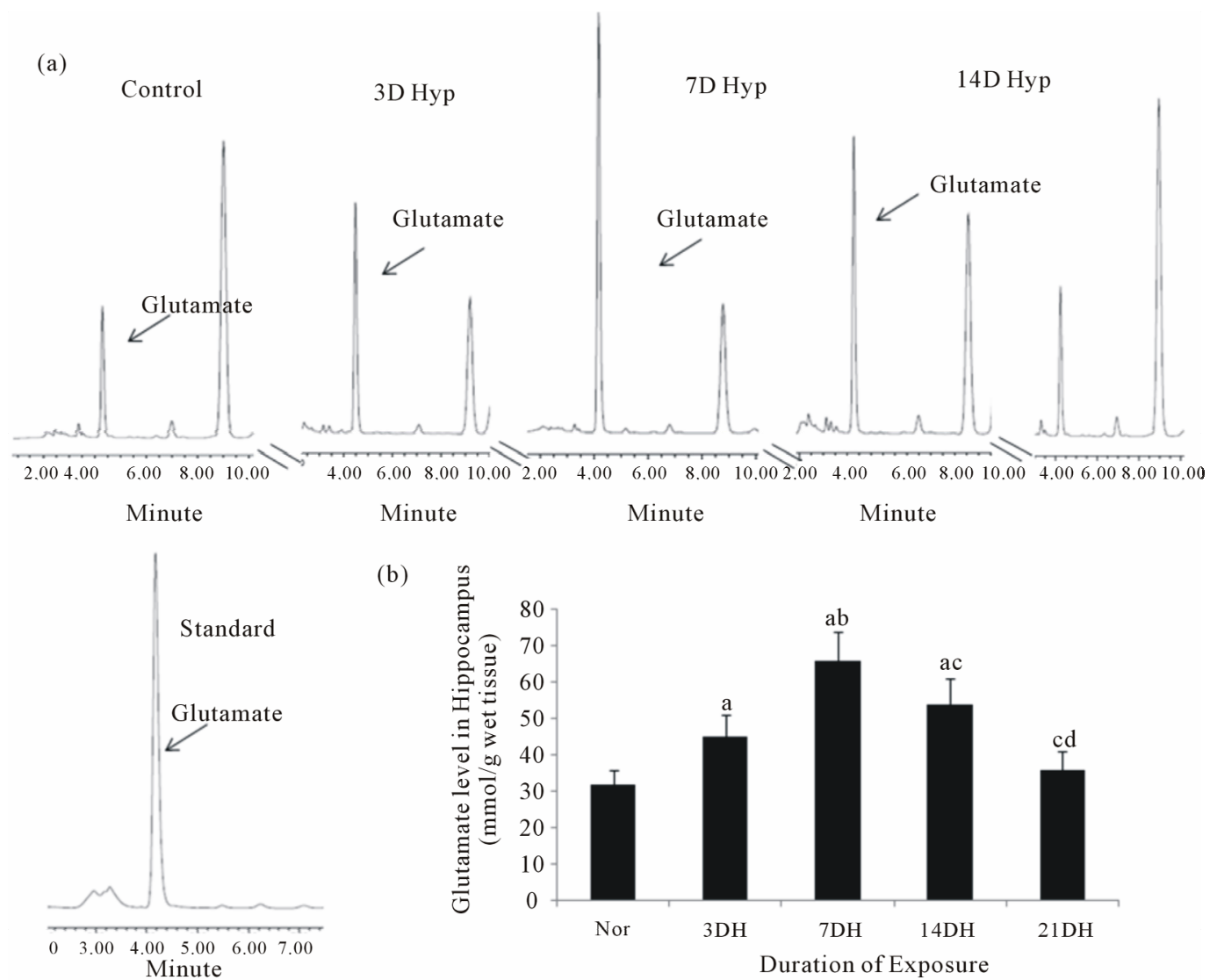

(b)

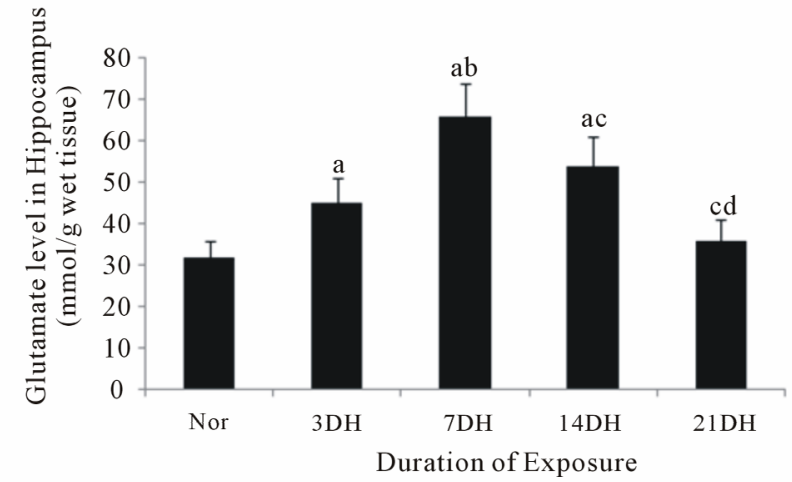

Figure 5. Representative HPLC Chromatograms showing the peak for Glutamate. (a) The peak for glutamate of standard, normoxic group, 3 days hypoxic group, 7 days hypoxic group, 14 days hypoxic group and 21 days hypoxic group. (b) Changes in the level of glutamate in hippocampus following exposure to HH. "a" denotes $p<0.05$ vs when compared with normoxia, "b" denotes $p<0.05$ vs when compared with 3 days hypoxia, "c" denotes $p<0.05$ Vs when compared to 7 days hypoxia and "d" denotes $p<0.05$ Vs when compared with 14 days hypoxia.

activity of rats but drastically reduces the exploratory behavior. However, this effect tends to normalize on prolonged exposure to $\mathrm{HH}$ for 21 days indicating the effect to be transient in nature.

In addition, rota rod test showed no significant change in the latency of fall following exposure to $\mathrm{HH}$. The rota rod test results confirm the observations seen in open field test and elevated plus maze that the chronic exposure to HH doesnot affects the locomotory activity in the rats. Chronic exposures for 21 days, however, showed no symptoms of anxiety in rats. The improvement in mood state on prolonged exposure may be a result of onset of acclimatization to hypobaric hypoxic condition. Supporting the present findings, there are several studies showing similar acclimatization to mild stress on exposure for prolonged period which depend on type, intensities and duration of stress exposure. However, studies in other acute stress model such as restraint and chronic unpredictable mild stress (CUMS) induces anxiety-like behaviors which leads to depressive mood state on prolonged exposure [27-29]. Unpredictable chronic mild stress results in depression like behavior in rats and has been used as a well accepted model to study molecular mechanisms underlying depressive disorders [25].

Exposure to stress can induce loss of interest in novel tasks which reveals the anhedonic behavior in rats. Anhedonia is a hallmark symptom found in depressed patients and frequently used as a behavioral marker for depressive disorders [30]. In the present study, drastic reduction in sucrose solution intake was noted on initial exposure to $\mathrm{HH}$ which persists for $14^{\text {th }}$ day followed by a gradual increase on prolonged exposure. The reduced solution intake could be because of induction of anhedonia in rats following exposure to hypoxia. Alternative explanation to the reduced sucrose solution intake could be induction of hypophagia following exposure to $\mathrm{HH}$ [6]. Thus, anhedonia could be a cause of hypophagia which in turn can induce loss of food intake and consequent decrease in body weight. Decrease in serotonin level on initial exposure to HH further indicates the possibility of anhedonia in rats [31]. However, the gradual increase in sucrose solution intake following $14^{\text {th }}$ day of hypoxic exposure indicates the onset of acclimatization process which reduced anhedonic behavior in rats. 
Does prolonged exposure to $\mathrm{HH}$ induce depression in rats? There are several reports that chronic exposure to restraint stress for 15 days ( 2 hrs daily) leads to the induction of depressive symptoms in rat $[32,33]$. Chronic injection of corticosterone at an elevated dose has been used in development of stress induced depression model in number of anti depressant studies [34]. Previous studies in hypoxic models also have reported the induction of anxiety-like behaviors in humans $[35,36]$. Chronic anxiety may in turn leads to the induction of depression in human as well as many animal models [37]. In the present studies, we assess the depressive symptom in rats using forced swim test following exposure to different duration of HH. Though forced swim test as well as the tail suspension test was initially developed as behavioral screening tools for anti-depressant drug efficacy, these two tests are increasingly being used to measure a specific depression like behavior, behavioral despair, independent of any anti-depressant treatment [38-40]. The initial increase in immobility time in forced swim test indicates altered mood state following exposure to $\mathrm{HH}$ for 3 and 7 days. Since forced swim test itself can induce depression in rats. It is not clear whether increase in despair behaviors is due to exhaustive nature of test itself or is a result of hypoxic exposure. Since forced swim test requires the rats to keep on swimming actively for five minutes, it makes the rats more exhausted. However, exposure to $\mathrm{HH}$ for 7 days has been reported to induce mitochondrial dysfunction and compromised neuronal energetic due to reduced availability of glucose as a result of hypoxia induced hypophagia [14]. Prolonged exposure for 21 days onset acclimatization to $\mathrm{HH}$ and increased food intake resulting in improved the neuronal energy status. The present observation finds support from previous report on duration dependent increase in hippocampal neurodegeneration following exposure to $\mathrm{HH}$ for 7 days with gradual decreases on prolonged exposure for 21 days [41]. Increase in proinflammatory cytokines and decreased neurotrophic factors level in brain also affect the anxiety-like behaviors in rats [42]. Neurotransmitters like serotonin, nor-epinephrine and stress hormone corticosterone undergoes a duration dependent alteration in brain on exposure to various stressors and play an important role in anxiety and depression like behaviors in rats [43]. However, behavioral assessment using forced swim test with increase in duration of exposure to $\mathrm{HH}$ showed decrease in despair behavior. This finding suggests that though there are temporal inductions of anxiety-like behavior in rats exposed to $\mathrm{HH}$ for 7 days, it does not lead to induction of depressive disorder on prolonged exposure. Supporting the present observation, previous reports showed that mood state alteration on exposure to $\mathrm{HH}$ depends on the altitude and is transient in nature [44].
Exposure to intermittent HH has been shown to promote hippocampal neurogenesis and can be implicated as therapeutics to cure depression like disorders [45]. Preconditioning using mild intermittent $\mathrm{HH}(360 \mathrm{mmHg}, 2 \mathrm{~h})$ prevents inescapable footshock induced HPA axis hyperactivity and increases resistance to psychoemotional stresses resulting in marked anxiolytic and antidepressant effects in rats $[46,47]$. However, the longer duration of hypoxic exposure and extreme altitude results in opposing effects. While Shukitt-hale reported that acute exposure to $\mathrm{HH}$ induce alteration in mood state in human which get normalized within a small time window of 2 days [35], there are only few reports on the effect of chronic exposure to $\mathrm{HH}$ on mood state.

Neurotransmitters level as well as stress hormone alteration profoundly influence mood state of an organism. In fact, the alteration in neurotransmitters may be primary causative factor reflected at behavioral level. In agreement with previous reports, decreased hippocampal serotonin level on initial exposure to $\mathrm{HH}$ indicates the involvement of serotonegic system in causing behavioral alteration. Serotonin is a modulatory neurotransmitter and has widely been implicated in depressive symptomatic manifestation in many model organisms. Further studies on monoaminergic system and subsequent changes in their receptors need to be carried out to depict the molecular mechanism underlying this behavioral impairment.

The initial elevation of corticosterone level in hippocampus invokes adaptive response to hypoxic stress. However, prolonged elevated level of corticosterone for 7 days could cause mal-adaptation to hypoxic stress leading to induction of anxiety-like behavior in rats. Reduction in corticosterone level following chronic exposure for 21 days might result in acclimatization to hypoxic stress and subsequent elevation in mood state. In line with the previous reports, the present finding support the corticosterone hypothesis of depression which reports that persistent elevated corticosterone play a major role in induction of depression [48]. Recent observations from our laboratory also showed that persistent elevated corticosterone level in the hippocampal region of the brain aggravates $\mathrm{HH}$ induced neurodegeneration and memory impairment and optimal maintenance of corticosterone level in hippocampus resulted in improved memory function [15]. Moreover, neuronal loss and reduction in hippocampal volume in depressed patient may be correlated the elevated level of corticosterone in hippocampus. It has been reported that memory loss is closely associated with depressive disorders. On the other hand, duration dependent increase in glutamate level in hippocampus following exposure to $\mathrm{HH}$ may leads to induction of glutamate excitotoxicity [17]. Subsequent decrease in glutamate levels indicate the reduction in exci- 
totoxicity induced neurodegeneration following prolonged exposure to HH. Decrease in corticosterone and glutamate level explain improvement in mood state on prolonged exposure for 21 days and support behavioral data that despite initial induction of anxiety-like behavior, prolonged exposure to $\mathrm{HH}$ may not induce depression like behavior in rats.

\section{Conclusion}

Chronic exposure to $\mathrm{HH}$ for 7 days induces anxiety-like behaviors in rats. Prolonged exposure to HH for 21 days results in progressive improvement in behavioral pattern of rats indicating that high altitude induced anxiety-like behavior is transient in nature. Alteration in the levels of glutamate, serotonin and corticosterone might be involved in HH-induced anxiety-like behavior in rats and modulation of these factors could reverse the hypoxiainduced behavioral impairment. The findings may be helpful in developing proper acclimatization schedule which will enhance the human performance at high altitude to a considerable extent.

\section{Acknowledgements}

The study was supported by Defence Research and Development Organization, Ministry of Defence, Government of India. We are also thankful to Council of Scientific and Industrial Research, New Delhi for providing fellowship for conduction of the study smoothly.

\section{REFERENCES}

[1] R. W. Baumgartner, V. Eivhenberger and P. Bartsch, "Postural Ataxia at High Altitude Is Not Related to Mild to Moderate Acute Mountain Sickness," European Journal of Applied Physiology, Vol. 86, No. 4, 2002, pp. 322326. http://dx.doi.org/10.1007/s00421-001-0534-8

[2] D. M. Baily and B. Davies, "Acute Mountain Sickness, Prophylactic Benefits of Antioxidant Vitamin Supplementation at High Altitude," High Altitude Medicine Biology, Vol. 2, No. 1, 2001, pp. 21-29.

http://dx.doi.org/10.1089/152702901750067882

[3] X. Y. Li, X. Y. Wu, C. Fu, X. F. Shen, C. B. Yang and Y. H. Wu, "Effects of Acute Exposure to Mild or Moderate Hypoxia on Human Psychomotor Performance and Visual-Reaction Time," Space Medicine and Medical Engineering (Beijing), Vol. 13, No. 4, 2000, pp. 235-239. http://dx.doi.org/10.2165/00007256-199316020-00003

[4] M. Bahrke and B. S. Hale, "Effect of Altitude on Mood, Behavior and Cognitive Functioning," Sports Medicine, Vol. 16, 1993, pp. 97-125.

[5] A. J. Hamilton, L. A. Trad and A. Cymerman, "Alterations in Human Upper Extremity Motor Function during Acute Exposure to Simulated Altitude," Aviation and Space Environmental Medicine, Vol. 62, 1991, pp.759764.
[6] S. B. Singh and W. Selvamurthy, "Effect of Intermittent Chronic Exposure to Hypoxia on Feeding Behavior of Rats," International Journal of Biometeorology, Vol. 37, No. 4, 1993, pp. 200-202. http://dx.doi.org/10.1007/BF01387523

[7] L. Thakur, K. Ray, J. P. Anand and U. Panjwani, "Event Related Potential (ERP) P300 after 6 Months Residence at 4115 Meter," Indian Journal of Medical Research, Vol. 134, No. 1, 2011, pp. 113-117.

[8] S. Muthuraju, P. Maiti, P. Solanki, A. K. Sharma, S. B. Singh, D. Prasad and G. Ilavazhagan, "Cholinesterase Inhibitors Ameliorate Spatial Learning Deficits in Rats Following HH," Experimental Brain Research, Vol. 203, No. 3, 2010, pp. 583-592. http://dx.doi.org/10.1007/s00221-010-2266-7

[9] K. Barhwal, S. K. Hota, I. Baitharu, D. Prasad, S. B. Singh and G. Ilavazhagan, "Isradipine Antagonizes HH Induced CA1 Damage and Memory Impairment: Complementary hotaroles of L-Type Calcium Channel and NMDA Receptors," Neurobiology of Disease, Vol. 34, No. 2, 2009, pp. 230-244. http://dx.doi.org/10.1016/j.nbd.2009.01.008

[10] V. Krishnan and E. J. Nestler, "The Molecular Neurobiology of Depression," Nature, Vol. 455, 2008, pp. 894902. http://dx.doi.org/10.1038/nature07455

[11] P. L. Delgado, "Depression: The Case for a Monoamine Deficiency," Journal of Clinical Psychiatry, Vol. 6, 2006, pp. 7-11.

[12] C. Alexandre, D. Popa, V. Fabre, S. Bouali, P. Venault, K. P. Lesch, M. Hamon and J. Adrien, "Early Life Blockade of 5-Hydroxytryptamine 1A Receptors Normalizes Sleep and Depression-Like Behavior in Adult Knock-Out Mice Lacking the Serotonin Transporter," Journal of Neuroscience, Vol. 26, No. 20, 2006, pp. 55545564. http://dx.doi.org/10.1523/JNEUROSCI.5156-05.2006

[13] E. I. Flandreau, K. J. Ressler, M. J. Owens and C. B. Nemeroff, "Chronic Overexpression of Corticotropin-Releasing Factor from the Central Amygdala Produces HPA Axis Hyperactivity and Behavioral Anxiety Associated with Gene-Expression Changes in the Hippocampus and Paraventricular Nucleus of the Hypothalamus," Psychoneuroendocrinology, Vol. 37, No. 1, 2012, pp. 27-38. http://dx.doi.org/10.1016/j.psyneuen.2011.04.014

[14] I. Baitharu, S. N. Deep, V. Jain, K. Barhwal, A. S. Malhotra, S. K. Hota, D. Prasad and G. Ilavazhagan, "Corticosterone Synthesis Inhibitor Metyrapone Ameliorates Chronic Hypobaric Hypoxia Induced Memory Impairment in Rat," Behavioral Brain Research, Vol. 228, No. 1, 2012, pp. 53-65. http://dx.doi.org/10.1016/j.bbr.2011.11.030

[15] I. Baitharu, S. N. Deep, V. Jain, D. Prasad and G. Ilavazhagan, "Inhibition of Glucocorticoid Receptors Ameliorates Hypobaric Hypoxia Induced Memory Impairment in Rat," Behavioral Brain Research, Vol. 240, 2013, pp. 76-86. http://dx.doi.org/10.1016/j.bbr.2012.11.005

[16] W. N. Marsden, "Stressor-Induced NMDAR dysfunction as a Unifying Hypothesis for the Aetiology, Pathogenesis and Comorbidity of Clinical Depression," Medical Hy- 
potheses, 2011, p. 7.

[17] S. K. Hota, K. Barhwal, I. Baitharu, D. Prasad, S. B. Singh and G. Ilavazhagan, "Bacopa Monniera Leaf Extract Ameliorates Hypobaric Hypoxia Induced Spatial Memory Impairment," Neurobiology of Disease. Vol. 34, No. 1, 2009, pp. 23-39.

http://dx.doi.org/10.1016/j.nbd.2008.12.006

[18] A. V. Kalueff, T. Keisala, A. Minasyan, M. Kuuslahti and P. Tuohimaa, "Temporal Stability of Novelty Exploration in Mice Exposed to Different Open Field Tests," Behavioral Processes, Vol. 72, No. 1, 2006, pp. 104-112.

[19] J. C. O'Connor, M. A. Lawson, C. Andre, M. Moreau, J. Lestage, N. Castanon, K. W. Kelley and R. Dantzer, "Lipopolysaccharide-Induced Depressive-Like Behavior Is Mediated by Indoleamine 2,3-Dioxygenase Activation in Mice," Molecular Psychiatry, Vol. 14, 2009, pp. 511522. http://dx.doi.org/10.1038/sj.mp.4002148

[20] Y. N. Wong, B. M. Chien and A. P. D'mello, “Analysis of Corticosterone in Rat Plasma by High-Performance Liquid Chromatography," Journal of Chromatography B: Biomedical Application, Vol. 661, No. 2, 1994, pp. 211218. http://dx.doi.org/10.1016/0378-4347(94)00346-7

[21] A. Mishra and K. P. Roy, "2-OHE 2 -Induced Oocyte Maturation Involves Steroidogenesis in Cat Fish," Journal of Endocrinology, Vol. 189, 2006, pp. 341-353. http://dx.doi.org/10.1677/joe.1.06686

[22] B. A. Gaudiano, D. Young, I. Chelminski and M. Zimmerman, "Depressive Symptom Profiles and Severity Patterns in Outpatients with Psychotic vs Nonpsychotic Major Depression," Comparative Psychiatry, Vol. 49, No. 5, 2008, pp. 421-429. http://dx.doi.org/10.1016/j.comppsych.2008.02.007

[23] G. M. Saletu-Zyhlarz, P. Anderer, O. Arnold and B. Saletu, "Confirmation of the Neurophysiologically Predicted Therapeutic Effects of Trazodone on Its Target Symptoms Depression, Anxiety and Insomnia by Postmarketing Clinical Studies with a Controlled-Release Formulation in Depressed Outpatients," Neuropsychobiology, Vol. 48, 2003, pp. 194-208. http://dx.doi.org/10.1159/000074638

[24] M. K. Sun and D. L. Alkon, "Induced Depressive Behavior Impairs Learning and Memory in Rats," Neuroscience, Vol. 129, No. 1, 2004, pp. 129-139. http://dx.doi.org/10.1016/j.neuroscience.2004.07.041

[25] C. Song, X. Y. Zhang and M. Manku, "Increased Phospholipase A2 Activity and Inflammatory Response But Decreased Nerve Growth Factor Expression in the Olfactory Bulbectomized Rat Model of Depression: Effects of Chronic Ethyl-Eicosapentaenoate Treatment," Journal of Neurosciences, Vol. 29, No. 1, 2009, pp. 14-22.

[26] R. J. Katz, K. A. Roth and B. J. Carroll, "Acute and Chronic Stress Effects on Open Field Activity in the Rat: Implications for a Model of Depression," Neuroscience and Biobehavioral Review, Vol. 5, No. 2, 1981, pp. 247251. http://dx.doi.org/10.1016/0149-7634(81)90005-1

[27] W. P. Paré and G. B. Glavin, "Restraint Stress in Biomedical Research: A Review," Neuroscience \& Biobehavioral Reviews, Vol. 10, No. 3, 1986, pp. 339-370. http://dx.doi.org/10.1016/0149-7634(86)90017-5
[28] C. Chotiwat and R. B. Harris, "Increased Anxiety-Like Behavior during the Post-Stress Period in Mice Exposed to Repeated Restraint Stress," Hormone and Behaviour, Vol. 50, No. 3, 2006, pp. 489-495.

http://dx.doi.org/10.1016/j.yhbeh.2006.06.007

[29] P. Willner, "Validity, Reliability and Utility of the Chronic mild Stress Model of Depression: A 10-Year Review and Evaluation," Psychopharmacology (Berl), Vol. 134, No. 4, 1997, pp. 319-329.

http://dx.doi.org/10.1007/s002130050456

[30] T. Strekalova, R. Spanagel, D. Bartsch, F. A. Henn and P. Gass, "Stress-Induced Anhedonia in Mice is Associated with Deficits in Forced Swimming and Exploration," Neuropsychopharmacology, Vol. 29, No. 11, 2004, pp. 2007 2017. http://dx.doi.org/10.1038/sj.npp.1300532

[31] S. Scheggi, G. Marchese, F. Borsini, F. Bordi and M. G. De Montis, "Effects of the 5-HT(6) Receptor Agonist ST 1936 on Depression- and Anhedonia-like Experimental Models," Behavioral Brain Research, Vol. 224, No. 1, 2011, pp. 35-43.

http://dx.doi.org/10.1016/j.bbr.2011.05.019

[32] E. Poleszak, P. Wlaź, E. Kedzierska, D. Nieoczym, E. Wyska, J. Szymura-Oleksiak, S. Fidecka, M. Radziwoń-Zaleska and G. Nowak, "Immobility Stress Induces Depression-Like Behavior in the Forced Swim Test in Mice: Effect of Magnesium and Imipramine," Pharmacology Report, Vol. 58, No. 5, 2006, pp. 746-752.

[33] S. Sevgi, M. Ozek and L. Eroglu, "L-NAME Prevents Anxiety-Like and Depression-Like Behavior in Rats Exposed to Restraint Stress," Methods Find Experimental Clinical Pharmacology, Vol. 28, No. 2, 2006, pp. 95-99. http://dx.doi.org/10.1358/mf.2006.28.2.977840

[34] Y. Zhao, R. Ma, J. Shen, H. Su, D. Xing and L. Du, "A Mouse Model of Depression Induced by Repeated Corticosterone Injections," European Journal of Pharmacology, Vol. 581, No. 1-2, 2008, pp. 113-120.

http://dx.doi.org/10.1016/j.ejphar.2007.12.005

[35] M. Nicolas, F. Thullier-Lestienne, C. Bouquet, B. Gardette, C. Gortan, F. Joulia, M. Bonnon, J. P. Richalet, P. Therme and J. H. Abraini, "An Anxiety, Personality and Altitude Symptomatology Study during a 31-day Period of Hypoxia in a Hypobaric Chamber (Experiment 'Everest-Comex 1997')," Journal of Environmental Psychology, Vol. 19, No. 4, 1999, pp. 407-414.

http://dx.doi.org/10.1006/jevp.1999.0139

[36] B. L. Shukitt and L. E. Banderet, "Mood States at 1600 and 4300 Meters Terrestrial Altitude," Aviation, Space and Environmental Medicine, Vol. 59, No. 6, 1988, pp. 530532.

[37] J. Fawcett and H. M. Kravitz, "Anxiety Syndromes and Their Relationship to Depressive Illness," Journal of Clinical Psychiatry, Vol. 44, No. 8, 1983, pp. 8-11.

[38] A. J. Dunn and A. H. Swiergiel, "Effects of Acute and Chronic Stressors and CRF in Rat and Mouse Tests for Depression," Annals of New York Academy of Sciences, Vol. 1148, 2008, pp. 118-126. http://dx.doi.org/10.1196/annals.1410.022

[39] M. Banasr and R. S. Duman, "Glial Loss in the Prefrontal Cortex Is Sufficient to Induce Depressive-Like Behaviors," 
Biological Psychiatry, Vol. 64, No. 10, 2008, pp. 863-870. http://dx.doi.org/10.1016/j.biopsych.2008.06.008

[40] E. A. O’Connor, E. P. Whitlock, T. L. Beil and B. N. Gaynes, "Screening for Depression in Adult Patients in Primary Care Settings: A Systematic Evidence Review," Annals of Internal Medicine, Vol. 151, No. 2009, pp. 793803. http://dx.doi.org/10.7326/0003-4819-151-11-200912010$\underline{00007}$

[41] P. Maiti, S. Muthuraju, G. Ilavazhagan and S. B. Singh, "Hypobaric Hypoxia Induces Dendritic Plasticity in Cortical and Hippocampal Pyramidal Neurons in Rat Brain," Behavioural Brain Research, Vol. 189, No. 2, 2008, pp. 233-243. http://dx.doi.org/10.1016/j.bbr.2008.01.007

[42] L. Song, W. Che, W. Min-Wei, Y. Murakami and K. Matsumoto, "Impairment of the Spatial Learning and Memory Induced by Learned Helplessness and Chronic Mild Stress," Pharmacology Biochemistry and Behavior, Vol. 83, No. 2, 2006, pp. 186-193. http://dx.doi.org/10.1016/j.pbb.2006.01.004

[43] J. M. Gorman and J. M. Kent, "SSRIs and SNRIs: Broad Spectrum of Efficacy beyond Major Depression," Journal of Clinical Psychiatry, Vol. 60, No. 4, 1999, pp. 3338.

[44] B. Shukitt-Hale, L. E. Banderet and H. R. Lieberman, "Elevation-Dependent Symptom, Mood, and Performance Changes Produced by Exposure to Hypobaric Hypoxia," International Journal of Aviation Psychology, Vol. 8, No. 4, 1998, pp. 319-334. http://dx.doi.org/10.1207/s15327108ijap0804_1

[45] X. H. Zhu, H. C. Yan, J. Zhang, H. D. Qu, X. S. Qiu, L. Chen, S. J. Li, X. Cao, J. C. Bean, L. H. Chen, X. H. Qin, J. H., Liu, X. C. Bai, L. Mei and T. M. Gao, "Intermittent Hypoxia Promotes Hippocampal Neurogenesis and Produces Antidepressant-Like Effects in Adult Rats," Journal of Neuroscience, Vol. 30, No. 38, 2010, pp. 12653 12663. http://dx.doi.org/10.1523/JNEUROSCI.6414-09.2010

[46] E. Rybnikova, V. Mironova, S. Pivina, E. Tulkova, N. Ordyan, N. Nalivaeva, A. Turner and M. Samoilov, "Involvement of the Hypothalamic-Pituitary-Adrenal Axis in the Antidepressant-Like Effects of Mild Hypoxic Preconditioning in Rats," Psychoneuroendocrinology, Vol. 32, No. 7, 2007, pp. 813-823. http://dx.doi.org/10.1016/j.psyneuen.2007.05.010

[47] E. A. Rybnikova, M. O. Samoilov, V. I Mironova, E. I Tyul'kova, S. G. Pivina, L. A. Vataeva, N. E. Ordyan, E. Y. Abritalin and A. I. Kolchev, "The Possible Use of Hypoxic Preconditioning for the Prophylaxis of Post-Stress Depressive Episodes," Neuroscience and Behavioral Physiology, Vol. 38, No. 7, 2008, pp. 721-726. http://dx.doi.org/10.1007/s11055-008-9038-x

[48] N. Calvo, I. D. Martijena, V. A. Molina and M. Volosin, "Metyrapone Pretreatment Prevents the Behavioral and Neurochemical Sequel Induced by Stress," Brain Research, Vol. 800, No. 2, 1998, pp. 227-235. http://dx.doi.org/10.1016/S0006-8993(98)00515-0 